Ecología

\title{
Variación nictemeral de los ensamblajes de macrocrustáceos en una playa arenosa del centro-norte de Veracruz, México
}

\author{
Nictemeral variation of macrocrustacean assemblages in a sandy beach from north-central \\ Veracruz, Mexico
}

\author{
Arturo Rocha-Ramírez*, Rafael Chávez-López, Ingrid Antillón-Zaragoza \\ y Francisco Ali Fuentes-Mendoza \\ Laboratorio de Ecología, Facultad de Estudios Superiores Iztacala, Universidad Nacional Autónoma de México, Av. de Los Barrios 1, \\ Los Reyes Iztacala, 54090, Tlalnepantla, Estado de México, México
}

Recibido el 10 de abril de 2015; aceptado el 28 de agosto de 2015

Disponible en Internet el 26 de febrero de 2016

\begin{abstract}
Resumen
En este estudio se analizaron las variaciones espacio-temporales de la estructura del ensamblaje de macrocrustáceos de la playa arenosa de Villa Rica, Veracruz. Se tomaron en cuenta ciclos nictemerales relacionados con las fases lunares, el análisis multidimensional no-métrico mostró una tendencia a la estabilidad ambiental. En el ensamblaje de macrocrustáceos se reconocieron 9 especies, de estas, Emerita benedicti, Excirolana braziliensis, Excirolana sp. y Pseudohaustorius sp. resultaron dominantes. Los valores más altos de densidad total, riqueza de especies y diversidad se registraron durante las mareas altas, las especies de isópodos cirolánidos y Emerita benedicti se distribuyeron en la zona inferior no saturada de la playa, Pseudohaustorius sp. se encontró en la zona media. El ensamblaje de macrocrustáceos es simple, con riqueza específica baja (3-5) y valores de diversidad bajos, con diferencias significativas durante el tiempo de estudio; sin embargo, la complejidad de la estructura del ensamblaje tendió a mantenerse en el tiempo.

Derechos Reservados ( 2015 Universidad Nacional Autónoma de México, Instituto de Biología. Este es un artículo de acceso abierto distribuido bajo los términos de la Licencia Creative Commons CC BY-NC-ND 4.0.
\end{abstract}

Palabras clave: Macrocrustáceos; Diversidad; Playa disipativa; Zona intermareal

\begin{abstract}
In this study, the structure of spatio-temporal variations of macrocrustacean assemblage were analyzed from the sandy beaches of Villa Rica, Veracruz. The nictemeral cycles related to lunar phases were taken into account; the non-metric multidimensional analysis showed an environmental stability trend. Within the macrocrustacean assemblages 9 species were recognized, of these, Emerita benedicti, Excirolana braziliensis, Excirolana sp. and Pseudohaustorius sp., were the dominant ones. The highest values of total density, species richness, and diversity were recorded during high tides. The cirolanid isopods and Emerita benedicti occurred in the lower unsaturated zone of the beach, and Pseudohaustorius sp. was found in the middle area. Macrocrustacean assemblage is simple, with low species richness (3-5) and low diversity values with significant differences throughout the study time; however, the assemblage structure complexity tended to be constant throughout time.

All Rights Reserved (C 2015 Universidad Nacional Autónoma de México, Instituto de Biología. This is an open access item distributed under the Creative Commons CC License BY-NC-ND 4.0.

Keywords: Macrocrustaceans; Diversity; Dissipative beach; Intertidal zone
\end{abstract}

\footnotetext{
* Autor para correspondencia.

Correo electrónico: arocha@unam.mx (A. Rocha-Ramírez).

La revisión por pares es responsabilidad de la Universidad Nacional Autónoma de México.
}

\section{Introducción}

La zona mesolitoral de las playas arenosas constituye el hábitat de gran diversidad de organismos, conformando ensamblajes complejos de macroinvertebrados (Borzone, Souza y Soares, 1996); en ellos, los crustáceos, moluscos y gusanos poliquetos 
habitualmente son los grupos dominantes (Defeo et al., 2009). Los crustáceos más representativos incluyen especies de Peracarida (Isopoda y Amphipoda) y Decapoda (Caridea y Anomura); en general, están representados por pocas especies, todas ellas altamente especializadas, con gran movilidad a lo largo de la playa (Ocaña et al., 2012). Desempeñan un papel importante en la regeneración de nutrientes (San Vicente y Sorbe, 1999) y conforman el grupo de depredadores secundarios en las cadenas tróficas marinas, vinculando a los productores con los consumidores terciarios (González et al., 2006). Debido a su tamaño y al poseer pocas defensas, representan un grupo de producción, densidad y vulnerabilidad alta, por lo que otros grupos encuentran en ellos una fuente alimentaria importante (González y Thiel, 2004).

La morfodinámica, composición bioquímica, contenido de materia orgánica, subsidios de detritos, tamaño medio del grano de la arena, mezcla de sedimentos y ancho de la zona intermareal constituyen los factores clave explicativos de la distribución vertical, densidad y estructura de los ensamblajes de macroinvertebrados (Brazeiro, Rozbaczylo y Fariña, 1998; Ramírez, 2012). Por otra parte, las oscilaciones periódicas de la distribución de la fauna también pueden ser explicadas por los ciclos de luz/oscuridad, las condiciones de marea y sus ciclos de vida, que en su mayoría involucran una fase larval planctónica, que es afectada por los procesos de transporte, dispersión y reclutamiento a lo largo de la línea de costa (Defeo, 1996).

La información con la que se cuenta actualmente es relativamente escasa, debido al reciente desarrollo de un cuerpo teórico para estos ecosistemas (McLachlan y Brown, 2006); sin embargo, para algunas especies de isópodos cirolánidos y de anomuros hippidos se ha documentado la relación con la marea
(Beyst, Vanaverbeke, Vincx y Mees, 2002), la agitación mecánica (Forward, Thaler y Singer, 2007), la migración nocturna y con los ritmos de actividad (Jaramillo, Dugan y Contreras, 2000).

A pesar de que México posee una longitud de línea de costa de $11,122 \mathrm{~km}$, de los cuales aproximadamente $3,000 \mathrm{~km}$ corresponden al golfo de México (Inegi, 2003), solo 7 investigaciones se han registrado para localidades mexicanas, 4 para el estado de Veracruz (Martínez, 2013; Méndez, Solis-Weiss y CarranzaEdwards, 1985; Miranda, 2012; Pacheco, 2010) y 3 para Baja California Sur (Angeloni, 2003; Cupul-Magaña y Téllez-Duarte, 1997; Ramírez, 2012); ninguna de ellas consideró estudios nictemerales o movimientos mareales en este tipo de hábitats. El objetivo de este estudio fue analizar la variación de los ensamblajes de crustáceos en ciclos nictemerales en la zona intermareal en diferentes fases lunares y su relación con las variables ambientales de la playa arenosa Villa Rica, Veracruz.

\section{Materiales y métodos}

La playa Villa Rica se encuentra localizada en el municipio de Actopan, Veracruz, entre las coordenadas $19^{\circ} 40^{\prime} 18^{\prime \prime} \mathrm{N}$ y 96 23'42" O (fig. 1 A). Es una playa arenosa disipativa, relativamente estrecha (40-70 m), el ancho es mayor de $54 \mathrm{~m}$ durante la marea baja (Vidal, Losada, Medina y Losada, 1995). La berma es extensa y la zona de rompiente se presenta a mayor distancia de la costa; en época de nortes se observa una ligera erosión, el resto de esta zona siempre es estable. Presenta forma cóncava y asimétrica hasta la boca de la laguna El Llano, y luego recta hasta llegar a la playa El Farallón. Está caracterizada por un trazo recto de $4 \mathrm{~km}$ y una orientación noroeste-sureste. El

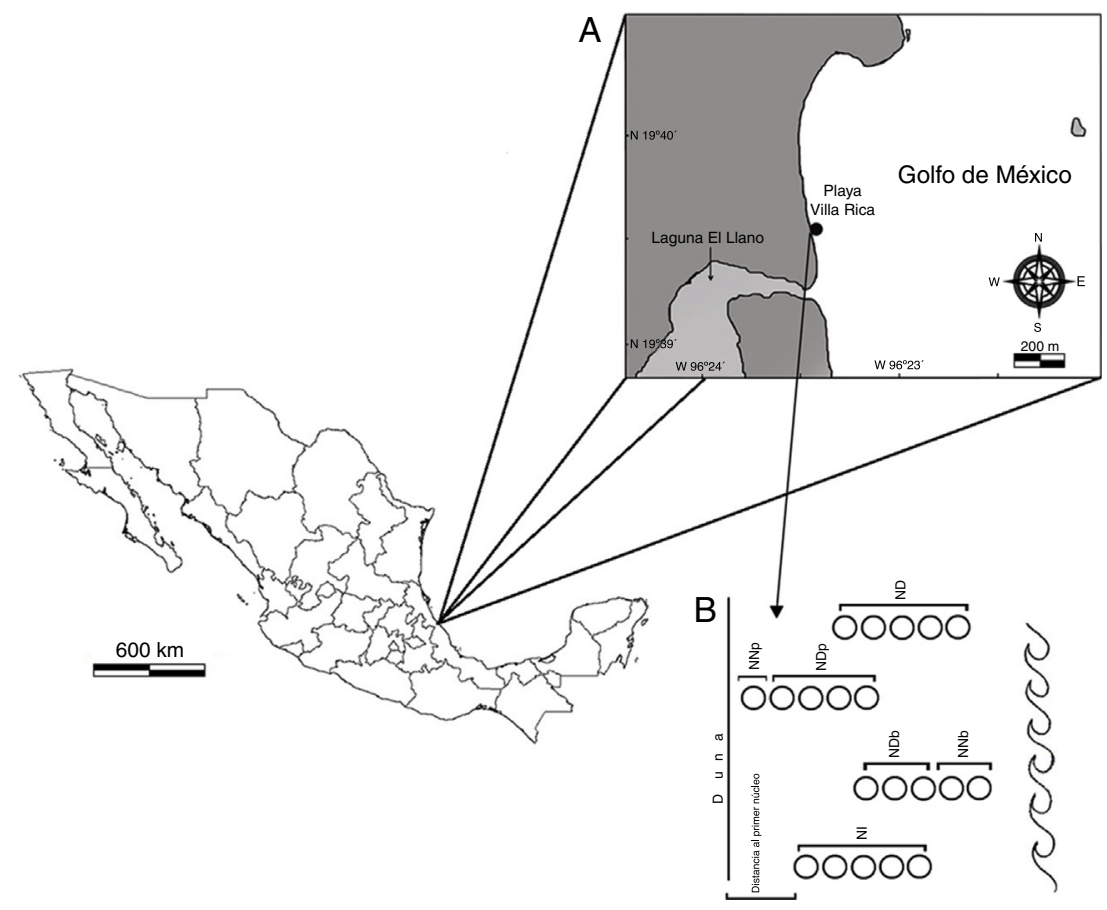

Figura 1. A. Ubicación geográfica del área de estudio. B. Ubicación de los núcleos extraídos de acuerdo con el momento de la marea.

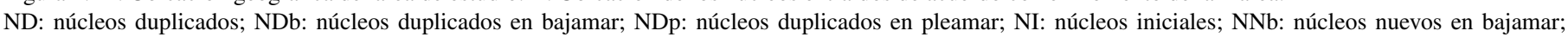
NNp: núcleos nuevos en pleamar. 
tipo de marea es mixta, predominantemente diurna y de poca amplitud (0.5-0.7 m) (Geissert, 1999). La zona supralitoral está delimitada por asentamientos humanos, en la porción inferior se encuentran parches pequeños del bejuco de playa (Ipomoea sp.) y en la zona mesolitoral el representante más conspicuo es el cangrejo fantasma (Ocypode quadrata).

De mayo de 2013 a marzo de 2014 se realizaron recolectas cada 2 meses con las siguientes fases lunares: mayo luna nueva (MayN), julio luna llena (JulLL), octubre cuarto menguante (OctM), diciembre luna nueva (DicN), febrero cuarto menguante (FebM) y marzo cuarto creciente (MarC). Se tomaron muestras cada $3 \mathrm{~h}$ durante un ciclo nictemeral. En la zona intermareal se ubicaron 2 transectos perpendiculares a la línea de costa, separados $10 \mathrm{~m}$ entre sí; en cada uno se tomaron 5 muestras de arena para analizar la textura y el contenido de materia orgánica por los métodos de Folk y Ward (1957) y Walkley y Black (1934) (en Muñoz, Mendoza, López, Hernández y Soler, 2001), respectivamente. Simultáneamente se registraron los datos para el cálculo de la pendiente de la playa mediante el método de Emery (1961) y los siguientes parámetros ambientales: temperatura del agua y arena - termómetro de suelo Oakton ${ }^{\circ} \mathrm{C}$ - , compactación de la arena — dinamómetro Alfa T-304, kg $\mathrm{cm}^{-2}$ - velocidad del viento (medidor de flujo Flowatch JDC, $\mathrm{m} \mathrm{s}^{-1}$ ), salinidad (refractómetro Ups Vista A366ATC), fuerza de la ola - dinamómetro modificado Alfa T-304, $\mathrm{kg} \mathrm{cm}^{-2}$ —, ancho de la zona de lavado y la amplitud de la marea (tabla de mareas de la Secretaría de Marina; Semar, 2013). En el tratamiento de datos a todos los valores de la amplitud de la marea se les sumó $0.3 \mathrm{~m}$ para evitar valores negativos.

En los mismos puntos de los transectos se tomaron muestras con un nucleador de PVC, con diámetro de $15.5 \mathrm{~cm}$, introducido a $20 \mathrm{~cm}$ de profundidad; la muestra obtenida se cernió en una malla de $0.5 \mathrm{~mm}$ de abertura, la arena restante se depositó en bolsas de plástico, con la etiqueta correspondiente y se fijaron con alcohol etílico al $96 \%$. En algunas recolectas los transectos se movieron de acuerdo con el momento de marea; a los núcleos que se extrajeron al inicio de cada ciclo (fig. 1 B) se les denominó núcleos iniciales (NI); a los que se extrajeron en el mismo sitio en relación con la hora anterior núcleos duplicados (ND) en pleamar (NDp) y en bajamar (NDb); los que se extrajeron en un sitio nuevo se les denominó núcleos nuevos en pleamar (NNp) y núcleos nuevos en bajamar ( $\mathrm{NNb}$ ). Adicionalmente, se tomaron 3 núcleos extra en la zona anterior a los transectos, generalmente se ubicaron en la zona no saturada.

Los organismos fueron separados de la totalidad de la muestra y se identificaron hasta el nivel taxonómico más bajo posible, utilizando los criterios de: Heard (1982); Kensley y Schotte (1989); Smith (1964) y Smith (1977). Posteriormente, se depositarán en la Colección Nacional de Crustáceos de la Universidad Nacional Autónoma de México.

\section{Tratamiento de datos}

A los datos de parámetros ambientales y biológicos se les probó la normalidad con la prueba W de Shapiro-Wilk $(\alpha=0.05)$ y posteriormente se aplicó la prueba de Kolmogorov-Smirnov $(\alpha=0.05)$ para examinar las diferencias entre los registros entre horas de muestreo y las fases lunares. Con los parámetros ambientales se realizó un análisis box plot, no se eliminaron los datos atípicos. Los análisis se realizaron con el software Sigma Plot 11.0 (Systat Software Inc, en prensa, disponible en: www.sigmaplot.com). Con los datos estandarizados por el total de la muestra se estimaron los valores de similitud de Bray-Curtis, con las matrices resultantes se realizaron 2 plots de ordenación indirecta (EMNM), uno para los datos de parámetros ambientales y otro para biológicos. Estos análisis se realizaron utilizando el software Primer 6.1.6 (Clarke y Gorley, 2006).

Las densidades fueron estandarizadas a org $\mathrm{m}^{-2}$. Para las condiciones iniciales se cuantificó la riqueza de especies y se calculó la densidad total, promedio, máxima y mínima; los valores de diversidad con el índice Shannon-Wiener $\left(\mathrm{H}^{\prime}=\right.$ bits org $\left.{ }^{-1}\right)$. Todos los cálculos se realizaron con el software Primer 6.1.6 (Clarke y Gorley, 2006). Con la prueba de $t$ de Hutchenson (1970) se probaron las diferencias entre los valores de diversidad $(\alpha=0.05)$, utilizando el software Past (Hammer, Harpe y Ryan, 2001). Para conocer la relación de los parámetros ambientales con los crustáceos se realizaron análisis de correlación entre parámetros ambientales vs. densidad total y densidad de las especies dominantes $(\alpha=0.05)$, utilizando el software Sigma Plot 11.0 (Systat Software Inc, en prensa, www.sigmaplot.com).

\section{Resultados}

\section{Parámetros ambientales}

Las características físicas de la playa Villa Rica durante el tiempo de la investigación fueron las siguientes: el tamaño predominante de grano fue $<0.5 \mathrm{~mm}$, que corresponde a arenas medianas, con $0.34 \pm 0.15 \%$ de materia orgánica. La pendiente osciló de 0.03 a 0.1 grados, lo cual define la playa como casi plana. Las variaciones del resto de los parámetros ambientales se presentan en la figura 2, se indican las diferencias significativas — prueba de Kolmogorov-Smirnov; $\alpha=0.05$ - entre cada fase lunar. La comparación de los datos ambientales en los periodos de horas luz y oscuridad no mostraron diferencias significativas en ninguna de las fases lunares (prueba de Kolmogorov-Smirnov; $\mathrm{p}=0.05$ ).

El análisis de ordenación indirecta EMNM arrojó un valor de estrés de 0.03 , que corresponde a una ordenación buena; en el plot se diferenciaron 2 grupos con valores de similitud de $80 \%$ a 90\% (fig. 3): el grupo A lo conformaron MayND, MayNN, JulLLD y JulLLN; en estos se registraron valores mayores de distancia de la duna al primer núcleo (de 34.5 a $55.7 \mathrm{~m}$ ), de temperatura del agua (de 25.9 a $31.6{ }^{\circ} \mathrm{C}$ ) y de la arena (de 26.9 a $31.9^{\circ} \mathrm{C}$ ), y valores elevados de compactación (de 0.03 a $0.04 \mathrm{~kg}$ $\mathrm{cm}^{-2}$ ). El grupo B lo conformaron el resto de los meses; en este grupo se registraron los valores mayores de altura de la marea (de 0.6 a $0.8 \mathrm{~m}$ ), velocidad del viento (de 3.9 a $5.6 \mathrm{~m} \mathrm{~s}^{-1}$ ), valores de compactación bajos $\left(0.02\right.$ y $\left.0.03 \mathrm{~kg} \mathrm{~cm}^{-2}\right)$ y los valores menores de distancia de la duna al primer núcleo (de 17.8 a $27 \mathrm{~m}$ ), fuerza de la ola (de 0.06 a $0.09 \mathrm{~kg} \mathrm{~cm}^{-2}$ ) y temperatura del agua (de 24.3 a $\left.25.8^{\circ} \mathrm{C}\right)$ y de la arena $\left(23.8\right.$ a $\left.25.7^{\circ} \mathrm{C}\right)$. 

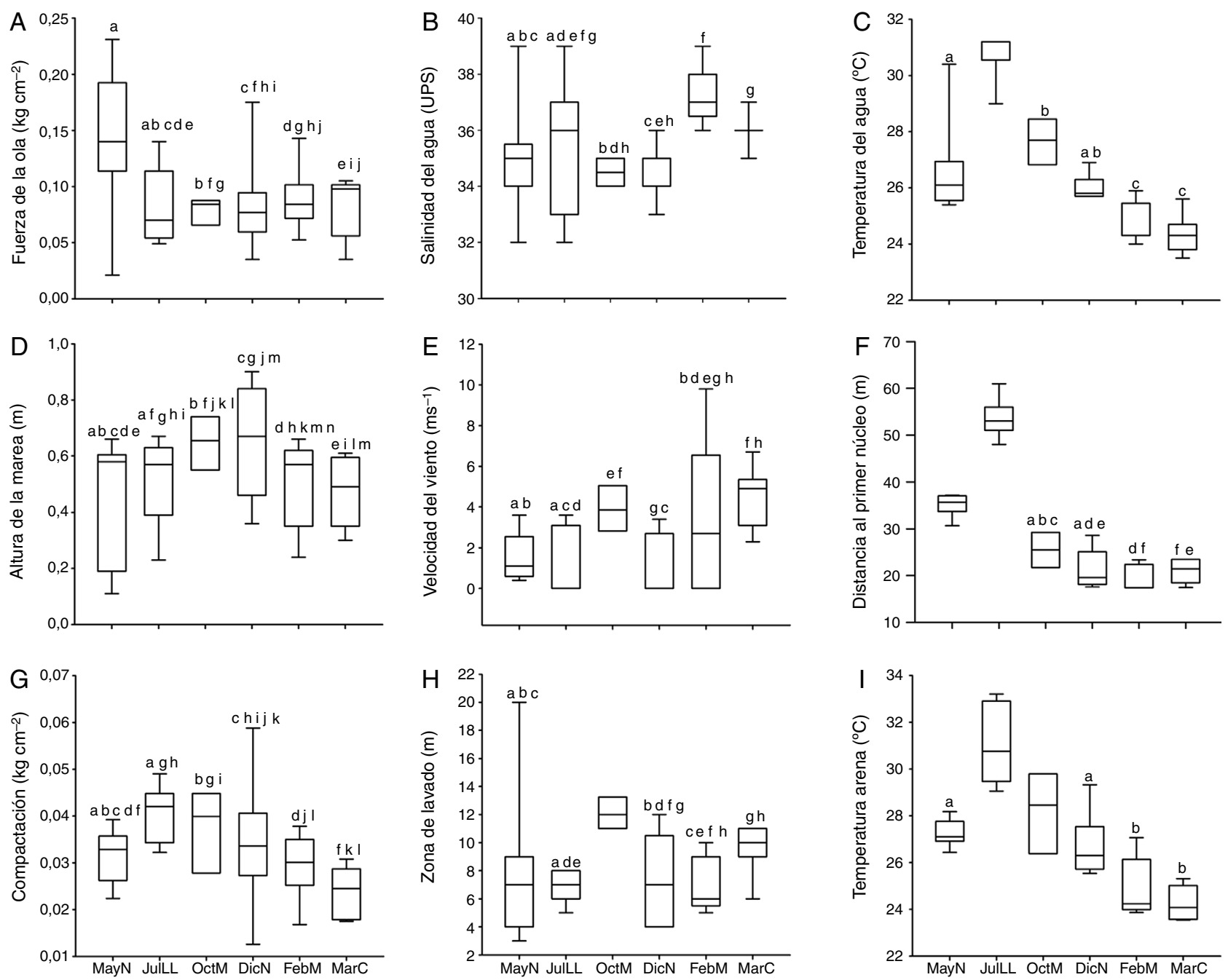

Figura 2. Box plots de los parámetros ambientales durante las diferentes fases lunares.

Las cajas con la misma letra no presentaron diferencias significativas de acuerdo con la prueba de Kolmogorov-Smirnov ( $\mathrm{p}>0.05$ ) (valores de altura de marea $-0.3 \mathrm{~m}$ ). DicN: diciembre luna nueva; FebM: febrero cuarto menguante; JulLL: julio luna llena,MarC: marzo cuarto creciente; MayN: mayo luna nueva; OctM: octubre cuarto menguante.

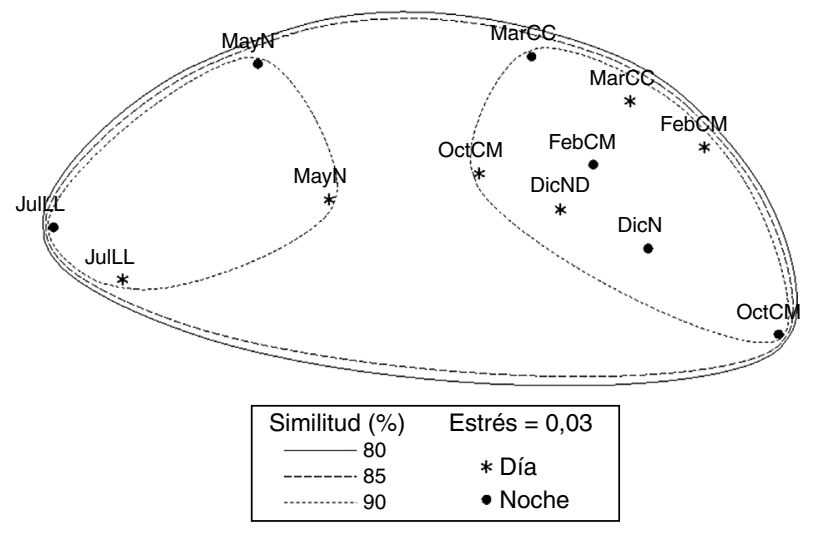

Figura 3. Plot del EMNM de parámetros ambientales en horas luz (D) y oscuridad (N) de la playa Villa Rica.

DicN: diciembre luna nueva; FebM: febrero cuarto menguante; JulLL: julio luna llena; MarC: marzo cuarto creciente; MayN: mayo luna nueva; OctM: octubre cuarto menguante.

\section{Variables ecológicas}

Los ensamblajes de crustáceos estuvieron representados por 9 especies: Albunea paretii (Guérin-Méneville, 1853), Ancinus jarocho Rocha-Ramírez, Chávez-López y Peláez-Rodríguez, 2010, Emerita benedicti Schmitt, 1935, Excirolana braziliensis Richardson, 1912, Excirolana sp., Pseudohaustorius sp., Lucifer sp., Caprellidae y Palaemonidae. La mayoría de los ejemplares de Emerita benedicti, Pseudohaustorius sp. y Albunea paretii fueron juveniles, mientras que los de Excirolana braziliensis y Excirolana sp. fueron juveniles y adultos.

La mayor riqueza de especies y los valores mayores de diversidad generalmente se registraron durante el periodo de horas luz, en JulLL y MarC se registró un valor de 1.96 y 1.67 bits org $^{-1}$, respectivamente, mientras que el valor menor se registró

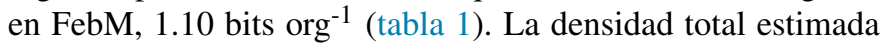
fue de 29,756 org $\mathrm{m}^{-2}$, Excirolana braziliensis contribuyó con $34 \%$, Excirolana sp. con $33 \%$ y Emerita benedicti con $24 \%$. En los momentos de pleamar, fundamentalmente en horas luz, se 
Tabla 1

Condiciones en los núcleos iniciales de la playa Villa Rica.

\begin{tabular}{|c|c|c|c|c|c|c|}
\hline & MayN & JulLL & OctM & $\operatorname{DicN}$ & FebM & MarC \\
\hline Riqueza de especies & 3.00 & 5.00 & 4.00 & 3.00 & 3.00 & 4.00 \\
\hline $\mathrm{H}^{\prime}\left(\right.$ bits org $\left.{ }^{-1}\right)$ & 1.37 & 1.96 & 1.35 & 1.28 & 1.10 & 1.67 \\
\hline Dominancia & 0.44 & 0.30 & 0.46 & 0.46 & 0.54 & 0.38 \\
\hline Densidad promedio & 14.72 & 73.44 & 135.43 & 70.66 & 20.71 & 64.77 \\
\hline Densidad total & 131.00 & 661.00 & 1217.00 & 636.00 & $1,086.00$ & 582.00 \\
\hline Densidad máxima & 79.00 & 291.00 & 715.00 & 371.00 & 742.00 & 318.00 \\
\hline Densidad mínima & 26.00 & 26.00 & 26.00 & 53.00 & 53.00 & 53.00 \\
\hline
\end{tabular}

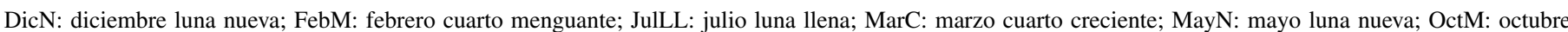
cuarto menguante.

registraron las mayores densidades totales; siendo Excirolana braziliensis $\left(2,014 \mathrm{org} \mathrm{m}^{-2}\right)$ y Excirolana $\mathrm{sp}$. (1,298 org $\left.\mathrm{m}^{-2}\right)$ las especies dominantes durante la pleamar en OctM y FebM, respectivamente, y Emerita benedicti durante bajamar de DicN $\left(424\right.$ org $\left.\mathrm{m}^{-2}\right)$.

Por otra parte, Excirolana braziliensis, Excirolana sp. y Emerita benedicti se presentaron durante todas las fases y en todos los núcleos extra, esta última solo se registró preferentemente en la parte inferior de la zona no saturada. Pseudohaustorius sp. y Albunea paretii no se registraron en DicN y en MayN, respectivamente. Ancinus jarocho se registró en horas de oscuridad en DicN, Lucifer sp. y Palaemonidae solo se registraron en las horas de oscuridad de OctM y Caprellidae en horas luz de FebM (fig. 4), con densidades muy bajas. Estas 4 taxa no se consideran habitantes de las playas arenosas.

Respecto a los núcleos NI (tabla 2), en JulLL se presentó la mayor riqueza de especies (5) y de diversidad (1.96 bits org ${ }^{-1}$ ), FebM presentó el valor más bajo de diversidad (1.07 bits org ${ }^{-1}$ )

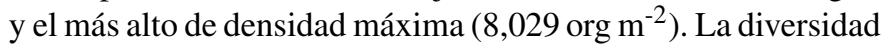
de los ensamblajes fue significativamente diferente al inicio de los muestreos - prueba de $t$ de Hutchenson, $\mathrm{p}<0.0005$ en todos los casos-, únicamente el valor de diversidad de OctM no fue diferente con respecto a los de MayN y DicN. Los valores más bajos de densidad mínima se presentaron en MayN, JulLL y OctM. Los valores más altos de densidad promedio y total se registraron en OctM y los más bajos en MayN. La correlación no fue significativa entre los parámetros ambientales vs. densidad total y de las especies dominantes.

El plot del EMNM de datos biológicos arrojó un valor de estrés de 0.12 , que corresponde a una ordenación buena. Se diferenciaron 4 grupos (fig. 5). El grupo 1 se conformó con valores de similitud de $70 \%$ a $80 \%$, FebMD y FebMN, en el que se registraron los valores más bajos de diversidad (1.07 y 1.09 bits org $^{-1}$ ) y dominancia (0.47 y 0.43), comparten 2 especies (Excirolana sp. y Excirolana braziliensis). El grupo 2 lo conformaron OctMD, OctMN y DicNN, con un valor de similitud de 70\%; en este grupo se registraron los valores mayores de riqueza de especies (5), valores de densidad altos $\left(4,715 ; 2,967\right.$ y 2,543 org $\left.\mathrm{m}^{-2}\right)$ $\mathrm{y}$ valores de diversidad intermedios $\left(1.65 ; 1.28 \mathrm{y} 1.74\right.$ bits org $\left.^{-1}\right)$ y dominancia (0.62; 0.48 y 0.67), comparten 3 especies (Emerita benedicti, Excirolana sp. y Excirolana braziliensis). El grupo 3 lo conformaron MarCD y MarCN, con un valor de similitud de $70 \%$, en este grupo se registraron valores de riqueza de especies (4-5), diversidad (1.79-1.82 bits org $\left.^{-1}\right)$ y dominancia altos (0.680. 76), comparten 4 especies (Emerita benedicti, Excirolana sp., Excirolana braziliensis y Pseudohaustorius sp.). El grupo 4 lo conformaron JulLLD y JulLLN, con valores de similitud de 70\% a $75 \%$, en el que se registraron los valores más altos de riqueza de especies (5), diversidad (1.72-2.11 bits org $^{-1}$ ) y dominancia (0.63-0.74), valores bajos de densidad (1,084-1,456 org $\left.\mathrm{m}^{-2}\right)$, además de compartir 5 especies (Emerita benedicti, Excirolana sp., Excirolana braziliensis, Pseudohaustorius sp. y Albunea paretii). En MayNN se registraron los valores más bajos de riqueza de especies (3) y densidad (344 org $\mathrm{m}^{-2}$ ). En MayND se registró un valor alto de diversidad (1.6 bits $\left.\mathrm{org}^{-1}\right)$, intermedio de riqueza de especies (4) y bajo de densidad $\left(1,429\right.$ org $\left.^{-2}\right)$.

En DicND se registraron valores intermedios de riqueza de especies (4) y densidad $\left(1,775 \mathrm{org} \mathrm{m}^{-2}\right)$, valor de diversidad bajo (1.13 bits org $^{-1}$ ) y el más bajo de dominancia (0.41). En JulLL, DicN, FebM y MarC las muestras se recolectaron en NI, ND,

Tabla 2

Porcentaje de contribución por tipo de núcleo y densidad por fase lunar de la playa Villa Rica, Veracruz.

\begin{tabular}{|c|c|c|c|c|c|c|c|c|c|c|c|c|}
\hline & \multicolumn{2}{|l|}{ MayN } & \multicolumn{2}{|l|}{ JulLL } & \multicolumn{2}{|l|}{ OctM } & \multicolumn{2}{|l|}{$\mathrm{DicN}$} & \multicolumn{2}{|l|}{ FebM } & \multicolumn{2}{|l|}{ MarC } \\
\hline & $\%$ & $\%$ & $\%$ & $\%$ & $\%$ & $\%$ & $\%$ & $\%$ & $\%$ & $\%$ & $\%$ & $\%$ \\
\hline & C.N. & C.D. & C.N. & C.D. & C.N. & C.D. & C.N. & C.D. & C.N. & C.D. & C.N. & C.D. \\
\hline NI & 11.11 & 7.46 & 11.11 & 26.04 & 16.67 & 15.86 & 11.11 & 14.91 & 11.11 & 13.53 & 11.11 & 10.68 \\
\hline $\mathrm{NDb}$ & 8.89 & 5.97 & 17.78 & 10.42 & 16.67 & 3.10 & 15.56 & 11.18 & 15.56 & 4.95 & 17.78 & 21.84 \\
\hline $\mathrm{NNb}$ & 8.89 & 7.46 & 28.89 & 22.92 & 33.33 & 12.76 & 20.00 & 8.07 & 17.78 & 7.59 & 20.00 & 11.17 \\
\hline NDp & 0.00 & 0.00 & 0.00 & 0.00 & 0.00 & 0.00 & 0.00 & 0.00 & 0.00 & 0.00 & 0.00 & 0.00 \\
\hline $\mathrm{NNp}$ & 4.44 & 29.85 & 0.00 & 0.00 & 16.67 & 38.62 & 0.00 & 0.00 & 0.00 & 0.00 & 0.00 & 0.00 \\
\hline ND & 62.22 & 49.25 & 42.22 & 40.63 & 16.67 & 29.66 & 53.33 & 65.83 & 55.56 & 73.93 & 51.11 & 56.31 \\
\hline
\end{tabular}

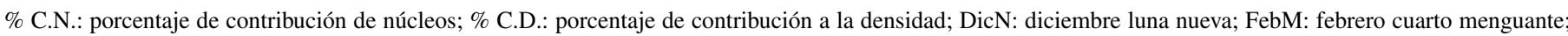

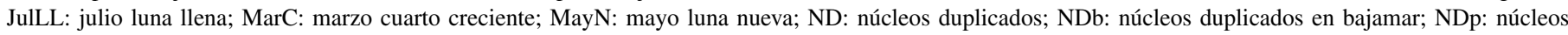
duplicados en pleamar; NI: núcleos iniciales; NNb: núcleos nuevos en bajamar; NNp: núcleos nuevos en pleamar; OctM: octubre cuarto menguante. 

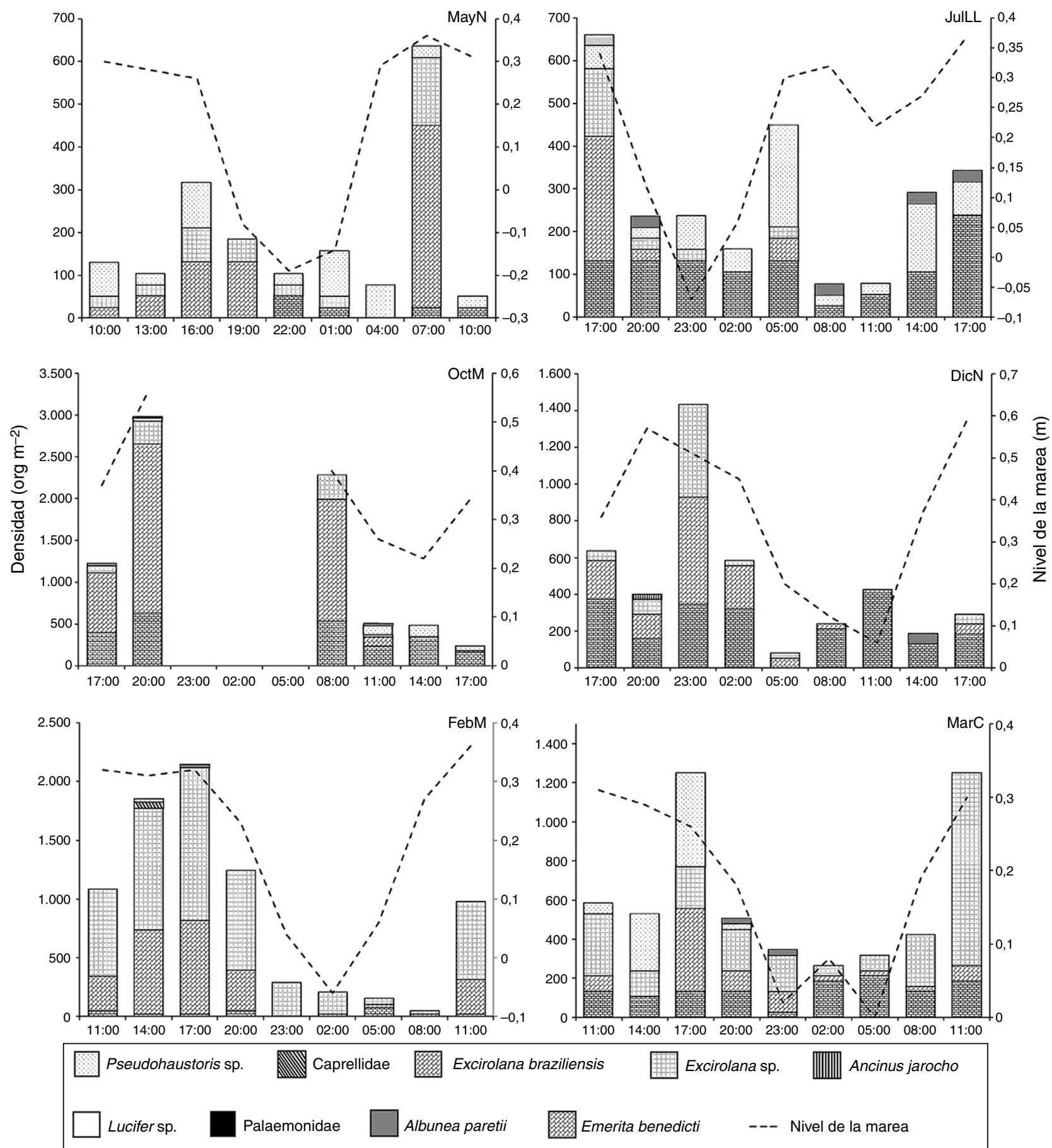

Figura 4. Densidad total de macrocrustáceos durante momentos de marea de un ciclo nictemeral en la playa Villa Rica.

DicN: diciembre luna nueva; FebM: febrero cuarto menguante; MarC: marzo cuarto creciente; MayN: mayo luna nueva; OctM: octubre cuarto menguante; JulLL: julio luna llena.y

$\mathrm{NDb}$ y NNb; durante OctM y JulLL en NI, ND NNp, NNb y $\mathrm{NDb}$ (tabla 2). En OctM el porcentaje máximo de contribución de núcleos fue por $\mathrm{NNb}$, en esta fase el ancho de la zona no saturada en la zona mesolitoral fue mayor (de 18 a $33 \mathrm{~m}$ ); $\sin$ embargo, el porcentaje máximo de contribución a la densidad total lo aportaron los NNp. Durante MayN, JulLL, DicN, FebM y MarC el máximo porcentaje de contribución de los núcleos y la densidad total fue por ND, debido a que durante estas fases el ancho de zona no saturada en la zona intermareal fue menor.
En general, todas las especies no exhibieron un patrón de distribución por talla a lo ancho de la playa. Los registros de densidades indicaron que Excirolana sp. se distribuyó preferentemente en la zona no saturada durante MayN (106 org m $\left.{ }^{-2}\right)$; en JulLL, OctM, DicN, FebM y MarC se distribuyó a lo largo del transecto. Las densidades mayores se registraron en la zona no saturada (79; 159,$424 ; 1,113$ y 556 org m$^{-2}$, respectivamente). Excirolana braziliensis se distribuyó a lo largo del transecto durante OctM, DicN y MarC, con mayores densidades en la 


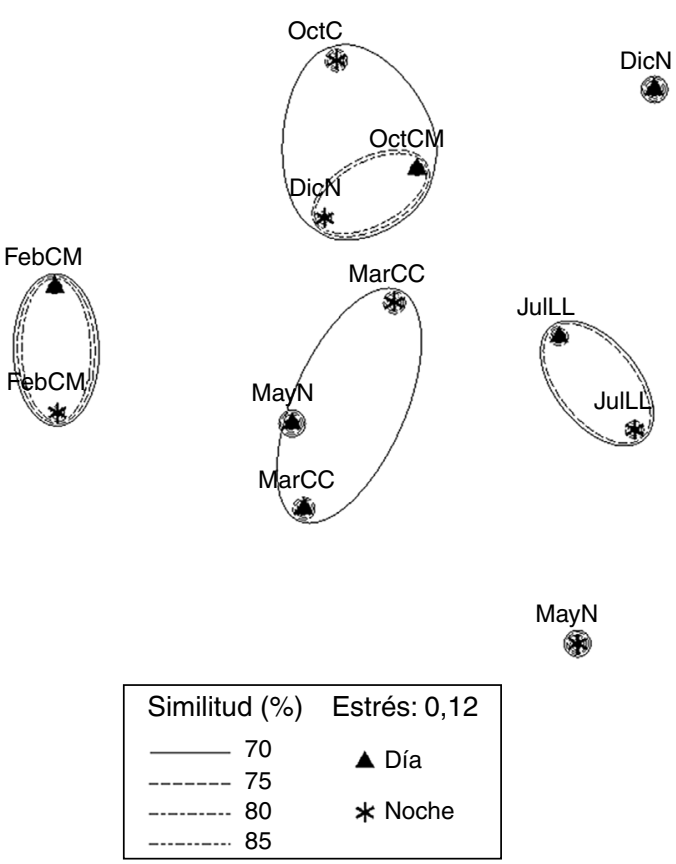

Figura 5. Plot del EMNM de las densidades en horas luz (D) y oscuridad (N) de macrocrustáceos de la playa Villa Rica.

DicN: diciembre luna nueva; FebM: febrero cuarto menguante; JulLL: julio luna llena; MarC: marzo cuarto creciente; MayN: mayo luna nueva; OctM: octubre cuarto menguantey.

zona no saturada $\left(901 ; 265\right.$ y $238 \mathrm{org} \mathrm{m}^{-2}$ ) y en la misma zona en MayN, JulLL y FebM (291; 106 y 530 org m$\left.^{-2}\right)$. Pseudohaustorius $\mathrm{sp}$. se presentó preferentemente en la franja de transición de la zona saturada y no saturada durante MayN y OctM (53 y 26 org m$\left.^{-2}\right)$, en la zona saturada durante $\operatorname{MarC}\left(256\right.$ org m$\left.^{-2}\right)$, en la zona no saturada durante FebM $\left(26 \mathrm{org} \mathrm{m}^{-2}\right)$ y a lo largo de todo el transecto en JulLL, con mayores densidades en la zona saturada ( $\left.132 \mathrm{org} \mathrm{m}^{-2}\right)$. Emerita benedicti se distribuyó a lo largo del transecto, presentó la mayor densidad en la zona no saturada durante JulLL, DicN y MarC (106; 344 y 106 org m$^{-2}$ ) la mayor densidad en la franja de transición durante OctM y MayN (238 y 26 org m$^{-2}$ ) y en FebM en la zona saturada $\left(26\right.$ org m $\left.{ }^{-2}\right)$.

\section{Discusión}

\section{Parámetros ambientales}

La variación en el perfil de la playa puede ser atribuida a cambios estacionales y pueden ocurrir alteraciones drásticas en las condiciones ambientales de la playa en pocas horas (Barros, Borzone y Rosso, 2001). Al respecto, en función del tamaño del grano medio de la arena, y por la pendiente casi plana, la playa Villa Rica puede ser considerada como una playa disipativa. La concentración de materia orgánica resultó ser baja, comparada con los valores registrados en playas de México (Ramírez, 2012) y de Cuba (Hernández, Contreras, Gallardo y Cancino, 1998). Sin embargo, McLachlan (1977) registró que la mayoría de las playas arenosas tienen concentraciones de materia orgánica bajas; es decir, entre $0.02 \%$ y $0.04 \%$ en playas de India. El régimen micromareal de la zona delimitó que la zona intermareal fuera estrecha, consecuentemente también sobre el ancho de la zona de lavado, que fue más pequeña en la época de nortes, al igual que la pendiente, debido al incremento del nivel del mar. De acuerdo con Salas-Monreal y Valle-Levinson (2008) puede incrementarse hasta $15 \mathrm{~cm}$ en esta época. El análisis de ordenación indirecta demostró que la zona presentó cierta tendencia a la estabilidad ambiental, al menos para los parámetros registrados.

\section{Variables ecológicas}

El sergéstido Lucifer sp., Ancinus jarocho, el palemónido y el caprélido se consideraron como fauna incidental, no son habitantes de las playas arenosas. Es así que solo 5 especies de crustáceos son habitantes de la playa Villa Rica y, de manera general, son reconocidas como propias de playas arenosas (McLachlan y Brown, 2006). La riqueza de especies es similar a la documentada por Pacheco (2010), quien registró 6 especies en playas del sur de Veracruz y menor a las documentadas por Méndez et al. (1985) en distintas playas del mismo estado, con 11 especies. Con respecto a playas con distintos estados morfodinámicos de otros países, la riqueza de especies es menor a la registrada por Ocaña et al. (2012) y mayor a la de Soares, Fernandes y Soares (2006).

La composición de los ensamblajes es simple, está integrada de 3 a 5 especies. Esta condición determinó que los valores de similitud fueran elevados en las diferentes fases lunares; al mismo tiempo, los de las horas luz y oscuridad. Por otra parte, la variación de la densidad de las especies determinó valores de diversidad relativamente bajos y significativamente diferentes; sin embargo, la complejidad de la estructura de los ensamblajes tiende a mantenerse temporalmente. Al respecto, McLachlan (1988) propuso que en playas expuestas y reflectivas la diversidad es controlada principalmente por el entorno físico; en consecuencia, en la playa Villa Rica los factores e interacciones biológicas pueden ser más importantes en la estructuración de los ensamblajes de macroinvertebrados (Defeo y McLachlan, 2005).

A pesar de que con anterioridad se extrajeron muestras de arena del mismo sitio en los núcleos ND, aun así se recolectaron organismos en horas posteriores, de tal manera que contribuyeron con un porcentaje elevado a la densidad total. Es posible que los organismos llegaran con las olas, lo que significa que son reclutados en la playa. Por otro lado, los organismos pudieron haber estado en las paredes adyacentes del núcleo vacío y por la acción mecánica del oleaje, la arena fue removida y depositada junto con ellos; esto confirmaría que son retenidos en esta zona.

Estudios comunitarios han demostrado que los crustáceos generalmente son dominantes en los niveles superiores de las playas; la mayor densidad se registró generalmente en los niveles superiores de la zona intermareal, lo que coincide con Dahl (1952), quien propuso que existe un patrón definido de distribución de la macrofauna asociada a playas arenosas, donde la abundancia de los individuos disminuye desde la zona litoral activa supra hacia los niveles inferiores de la zona mesolitoral, mientras que el número de especies aumenta en esa misma dirección. Es posible que los crustáceos presentes en playa Villa Rica representen las secciones alta y media de este esquema, la 
sección superior es habitada por los isópodos Excirolana braziliensis y Excirolana sp., la sección media dominada por el anfípodo Pseudohaustorius sp. y la parte inferior por los anomuros Albunea paretii y Emerita benedicti. Estos resultados también concuerdan con los de Haynes y Quinn (1995), quienes registraron en playas de Victoria, Australia, en donde los isópodos se limitaron a las zonas altas y los anfípodos a zonas bajas de la playa, con varios anomuros que habitan toda la zona mesolitoral.

Lepka (2008) señaló que algunas especies de cirolánidos suelen ser frecuentes en playas disipativas; al respecto, resultados similares a los de este estudio fueron observados para Excirolana braziliensis y Excirolana armata en playas arenosas micromareales de Uruguay (Yannicelli, Palacios y Giménez, 2001). Estas observaciones demuestran que durante el ciclo mareal los isópodos exhiben patrones de distribución en la playa, que están determinados por ritmos endógenos; regulando la capacidad natatoria de los isópodos (Naylor y Rejeki, 1996). En la parte superior de la playa, durante la pleamar, el movimiento del agua desencadena la natación, durante la bajamar el nado cesa y excavan en la arena, aunado a la capacidad de respirar aire, mientras que otros crustáceos están obligados a habitar la orilla inferior y dependen del agua; consecuentemente son retenidos en la playa.

Emerita talpoida se mueve de acuerdo con el movimiento de la marea a lo largo del día (Williams, 1984), también ocupa posiciones que coinciden con la profundidad de la zona de lavado para poder alimentarse (Jaramillo et al., 2000). En general, los hippidos adultos se distribuyen en el límite de la playa, mientras que los juveniles en la parte superior de la zona de lavado durante la bajamar. En la playa Villa Rica la mayoría de los ejemplares de Emerita benedicti fueron juveniles, se distribuyeron a lo largo de todo el segmento de la playa estudiado y probablemente se retiene en la zona no saturada durante la bajamar.

Los anfípodos son poco influenciados por el clima de la zona de lavado, y generalmente tienen un movimiento autónomo activo en los niveles superiores de la playa (Giménez y Yannicelli, 1997), aunque algunas especies son muy sensibles a la desecación (Méndez et al., 1985), por lo que la arena fina a mediana les permite mantenerse húmedos, moverse con mayor facilidad y excavar rápidamente. Lo anterior puede explicar la distribución de Pseudohaustorius sp. en toda la zona intermareal, y las densidades mayores en la zona saturada de la playa.

En este estudio es interesante notar que los crustáceos parecen moverse hacia la orilla de la playa durante la pleamar; esto sugiere que no se restringen a una franja estrecha $y$, por otra parte, pueden retenerse en ciertas áreas de la zona intermareal.

\section{Agradecimientos}

Agradecemos a numerosos estudiantes del módulo de Metodología Científica VI de la carrera de Biología de la FES Iztacala, UNAM, su asistencia en el trabajo de campo y de laboratorio. A los revisores anónimos que ayudaron a mejorar el manuscrito. Esta investigación fue apoyada parcialmente por la División de Investigación y por el Laboratorio de Ecología de la FES Iztacala, UNAM.

\section{Referencias}

Angeloni, C. P. E. (2003). Impacto del uso recreativo sobre la fauna macrobentónica de las playas arenosas de la bahía de La Paz. Tesis doctoral. Departamento de Pesquerías y Biología Marina, Instituto Politécnico Nacional.

Barros, F., Borzone, C. A. y Rosso, S. (2001). Macroinfauna of six beaches near Guaratuba Bay, Southern Brazil. Brazilian Archives of Biology and Technology, 44, 351-364

Beyst, B., Vanaverbeke, J., Vincx, M. y Mees, J. (2002). Tidal and diurnal periodicity in macrocrustaceans and demersal fish of an exposed sandy beach, with special emphasis on juvenile plaice Pleuronectes platessa. Marine Ecology Progress Series, 225, 263-274.

Borzone, C. A., Souza, J. R. B. y Soares, A. G. (1996). Morphodynamic influence on the structure of inter and subtidal macrofaunal communities of subtropical sandy beaches. Revista Chilena de Historia Natural, 69, 565-577.

Brazeiro, A., Rozbaczylo, N. y Fariña, J. M. (1998). Distribución espacial de la macrofauna en una playa expuesta de Chile central: efectos de la morfodinámica intermareal. Investigaciones Marinas Valparaíso, 26, 119-126.

Clarke, K. R. y Gorley, R. N. (2006). Primerv6. Usermanual/tutorial. Plymouth: PRIMER-E Ltd.

Cupul-Magaña, L. A. y Téllez-Duarte, M. A. (1997). Variaciones espaciotemporales de la fauna macrobentónica de una playa arenosa y su relación con los cambios del perfil de playa y el tamaño de grano de los sedimentos, en playa el pelícano, Baja California. Ciencias Marinas, 23, 419-434.

Dahl, E. (1952). Some aspects of the ecology and zonation of the fauna on sandy beaches. Oikos, 4, 27.

Defeo, O. (1996). Recruitment variability in sandy beach macroinfauna: much to learn yet. Revista Chilena de Historia Natural, 69, 615-630.

Defeo, O. y McLachlan, A. (2005). Patterns, processes and regulatory mechanisms in sandy beach macrofauna: a multiscale analysis. Marine Ecology Progress Series, 295, 1-20.

Defeo, O., McLachlan, A., Schoeman, D. S., Schlacher, T. A., Dugan, J., Jones, A., et al. (2009). Threats to sandy beach ecosystems: a review. Estuarine, Coastal and Shelf Science, 81, 1-12.

Emery, K. O. (1961). A simple method of measuring beach profiles. Limnology and Oceanography, 6, 90-93

Folk, R. L. y Ward, W. C. (1957). Brazos river bar: a study in the significance of grain size parameters. Journal of Sedimentary Petrology, 27, 3-26.

Forward, R. B., Jr., Thaler, A. D. y Singer, R. (2007). Entrainment of the activity rhythm of the mole crab Emerita talpoida. Journal of Experimental Marine Biology and Ecology, 341, 10-15.

Geissert, D. (1999). Regionalización geomorfológica del estado de Veracruz, investigaciones geográficas. Boletín del Instituto de Geografía, 40, $23-47$.

Giménez, L. y Yannicelli, B. (1997). Variability of zonation patterns in temperate microtidal Uruguayan beaches with different morphodynamic types. Marine Ecology, 160, 197-207.

González, E. y Thiel, M. (2004). Índice bibliográfico sobre biodiversidad acuática de Chile: crustáceos peracáridos (Crustacea). Ciencia y Tecnología del Mar, 27, 133-150.

González, E., Thiel, M., Haye, P., Bruce, N., Lowry, J. y Roccatagliata, D. (2006). Notebook of third International Course on Ecology and Taxonomy of Peracarida. Coquimbo: Universidad Católica de Chile.

Hammer, Ø., Harpe, D. A. T. y Ryan, P. D. (2001). PAST: Paleontological statistics software package for education and data analysis. Paleontología Electrónica, 4, 9 .

Haynes, D. y Quinn, G. P. (1995). Temporal and spatial variability in community structure of a sandy intertidal beach, Cape Patterson, Victoria, Australia. Marine and Freshwater Research, 46, 931-942.

Heard, R. W. (1982). Guide to common tidal marsh invertebrates of the northeastern Gulf of Mexico. Mississippi: Mississippi-Alabama Sea Grant Consortium MASGP-79-004.

Hernández, C., Contreras, S. H., Gallardo, J. A. y Cancino, J. M. (1998). Estructura comunitaria de la macroinfauna a lo largo de una playa arenosa de Chile central: Lenga, Bahía San Vicente. Revista Chilena de Historia Natural, 71, 303-311.

Hutchenson, K. (1970). A test for comparing diversities based on the Shannon formula. Journal of Theoretical Biology, 29, 151-154. 
Inegi (Instituto Nacional de Estadística y Geografía). (2003). Anuario estadístico de los Estados Unidos Mexicanos. Edición 2002. No. 61. Aguascalientes: Inegi.

Jaramillo, E., Dugan, J. y Contreras, H. (2000). Abundance, tidal movement, population structure and burrowing rate of Emerita analoga (Anomura, Hippidae) at a dissipative and reflective sandy beach in South central Chile. Marine Ecology, 21, 113-127.

Kensley, B. y Schotte, M. (1989). Guide to the marine isopod crustaceans of the Caribbean. Washington, D.C: Smithsonian Institution Press.

Lepka, D. L. (2008). Macrofauna de praias arenosas com diferentes graus de morfodinamismo no Parque Estadual da Ilha do Cardoso, SP, Brasil. Tesis de maestría. Programa de Pós-Graduação em Zoologia, Universidade Federal o Paraná.

Martínez, P. M. G.(2013). Morfodinámica y variación espacio-temporal de la fauna de cuatro playas arenosas del estado de Veracruz, México. Tesis. Facultad de Estudios Superiores Iztacala, Universidad Nacional Autónoma de México.

McLachlan, A. (1977). Studies on the psammolittoral meiofauna of Algoa Bay II. The distribution, composition and biomass of the meiofauna and macrofauna communities. Zoologica Africana, 12, 33-60.

McLachlan, A. (1988). Behavioural adaptations of sandy beach organisms: An ecological perspective. En G. Chelazzi y M. Vannini (Eds.), Behavioural adaptations to intertidal life (pp. 449-475). New York: Plenum Publishing Corporation.

McLachlan, A. y Brown, A. (2006). The ecology of sandy shores. Amsterdam: Academic Press Elsevier.

Méndez, U. M. N., Solis-Weiss, V. y Carranza-Edwards, A. (1985). La importancia de la granulometría en la distribución de organismos bentónicos. Estudio de playas del estado de Veracruz, México. Anales del Instituto de Ciencias del Mar y Limnología, 13, 45-56.

Miranda, M. M. (2012). Estructura de las comunidades de macroinvertebrados de la zona mesolitoral de playas arenosas del centro de Veracruz. México, D.F: Tesis. Facultad de Estudios Superiores Iztacala, Universidad Nacional Autónoma de México.

Muñoz, I. D., Mendoza, C. A., López, G. F., Hernández, M. M. y Soler, A. A. (2001). Manual de análisis de suelos: México. México, D.F: Escuela Nacional de Estudios Profesionales Iztacala, Universidad Nacional Autónoma de México.

Naylor, E. y Rejeki, S. (1996). Tidal migrations and rhythmic behavior of sandbeach Crustacea. Revista Chilena de Historia Natural, 69, 475-484.
Ocaña, F., Apín, Y., Cala, Y., Vega, A., Fernández, A. y Córdova, E. (2012). Distribución espacial de los macroinvertebrados de playas arenosas de Cuba oriental. Revista de Investigaciones Marinas, 32, 428-439.

Pacheco, R. S. (2010). Patrones de distribución de la macroinfauna en cuatro playas arenosas del estado de Veracruz, México. México, D.F: Tesis. Facultad de Estudios Superiores Iztacala, Universidad Nacional Autónoma de México.

Ramírez, T.R. A. (2012). Variación espacio-temporal de la comunidad macrobentónica del intermareal de la playa «El Conchalito», B.C.S., México. Tesis doctoral. La Paz: Centro Interdisciplinario de Ciencias Marinas, Instituto Politécnico Nacional.

Salas-Monreal, D. y Valle-Levinson, A. (2008). Sea-level slopes and volume fluxes produced by atmospheric forcing in estuaries: Chesapeake Bay case study. Journal of Coastal Research, 24, 208-217.

San Vicente, C. y Sorbe, J. C. (1999). Spatio-temporal structure of the suprabenthic community from Creixell beach (Western Mediterranean). Acta Oecologica, 20, 377-389.

Semar (Secretaría de Marina). (2013). Tabla de predicción de mareas [consultado Mar 2014]. Disponible en: http://www.semar.gob.mx/hidrocart/ grafnum/grafnum_tuxpan.htm

Smith, D. L. (1977). A guide to marine coastal plankton and marine invertebrate larvae. Iowa: Kendall/Hunt Publishing Co.

Smith, R. I. (1964). Keys to marine invertebrates of the Woods Hole Region: a manual for the identification of the more common marine invertebrates. Systematics-Ecology Program, Contribution No. 11. Woods Hole: Marine Biological Laboratory. Massachusetts.

Soares, R., Fernandes, R. y Soares, A. G. (2006). Community structure of macrobenthos in two tropical sandy beaches with different morphodynamic features, Rio de Janeiro, Brazil. Marine Ecology, 27, 160-169.

Systat Software Inc. SigmaPlot. Versión 11.0. San José, California, USA. Recuperado de: www.sigmaplot.com.

Vidal, C., Losada, M. A., Medina, R. y Losada, I. (1995). Modelos de morfodinámica de playas. Ingeniería del Agua, 2, 55-74.

Yannicelli, B., Palacios, R. y Giménez, L. (2001). Activity rhythms of two cirolanid isopods from an exposed microtidal sandy beach in Uruguay. Marine Biology, 138, 187-197.

Williams, A. B. (1984). Shrimps, lobsters and crabs of the Atlantic coast of Eastern United States, Maine to Florida. Washington, D.C: Smithsonian Institution Press. 